\title{
Single Channel Probe Utilizing the EGN Model to Estimate Link Parameters for Network Abstraction.
}

\author{
David J. Ives ${ }^{(1)}$, Hou-Man Chin ${ }^{(2)}$, F. J. Vaquero Caballero(1), Seb J. Savory ${ }^{(1)}$ \\ (1) University of Cambridge, 9 JJ Thomson Avenue, Cambridge, CB3 0FA, UK., di231@cam.ac.uk \\ ${ }^{(2)}$ Orange Polska, Al. Jerozolimskie 160, 02-326 Warsaw, Poland.
}

\begin{abstract}
We experimentally demonstrate multi-span link parameter abstraction using a single channel 11.5GBd probe. EGN based abstraction gave $\gamma=1.14 W^{-1} \cdot \mathrm{km}^{-1}$ c.f. $0.72 W^{-1} \cdot \mathrm{km}^{-1}$ for a GN based abstraction. The GN model overestimates the abstracted SNR by $0.4 d B$ c.f. the EGN model at $1000 \mathrm{~km}$.
\end{abstract}

\section{Introduction}

Wavelength routed transparent optical networks form the back bone of data transport networks and the Internet. To make the most effective use of the physical network resources the transmission signal is adapted to maximize the data rate over a given route ${ }^{1}$. The optimal adaptation of the signal and selection of wavelength and routing requires presents a complex management problem. The use of abstraction and virtualization allows the network management to be simplified making the allocation of resources more tractable. Abstraction reduces the complexity of physical components describing them as a few generic network elements with simplified properties. Virtualization provides control of these abstracted network elements which may exist as single physical components, aggregations of physical components or slices of physical components ${ }^{2}$.

In order to abstract the network it is necessary to obtain the properties of the physical components. We shall consider the coherent transmission paradigm where the transmission signal is impaired by stochastic noise and nonlinear interference from co-propagating channels. In ${ }^{3,4}$ continuous montioring of live signals is used to adapt the signal under network change. In $^{5}$ monitoring is used to update the abstract model to maintain the models accuracy.

In this paper we consider a new uncommissioned network that is to be abstracted. We sequentially probed the available links with a single transmission signal. The received symbol SNR is monitored as a function of launch power. From this we determine the ASE noise from the EDFA, the nonlinear parameter and the inherent noise of the transceiver. Given the ASE noise, nonlinear parameter and fiber transmission we abstract the optimal launch power for the link under full load and the worst case SNR degradation that can be expected.

\section{Measurements}

The measurements were made on a laboratory network test bed built around a Polatis 32×32 optical fiber switch. Connected to the switch are 10 fiber spans each consisting of $100 \mathrm{~km}$ of standard single mode fiber followed by an EDFA with gain $25 \mathrm{~dB}$ to compensate for the transmission and switch losses. Also connected to the switch are 4 more EDFA, an optical channel filter (with approximately 60 - $70 \mathrm{GHz}$ bandwidth), an OSA and a Ciena WaveLogic 2 coherent linecard capable of transmitting 40 Gbps at 11.5 GBaud using PMQPSK modulation.

The measurement consisted of measuring the transmission performance through 1 upto 10 fiber spans, 100 to $1000 \mathrm{~km}$, as a function of the launch signal power from -15 to $5 \mathrm{dBm}$. The experimental set up is shown in Fig.1. The transmitter output was first boosted by an EDFA and connected directly to the OSA. The attenuation required at the optical switch output to set the desired launch power was determined from the OSA trace after removing the ASE noise. The signal was launched into the first span at the desired launch power using the attenuation setting previously determined. The output of the first span was connected to the OSA and again the attenuation required at the optical switch output to set the desired launch power for the next span was determined from the OSA trace after removing the ASE noise. In this way the launch power into each

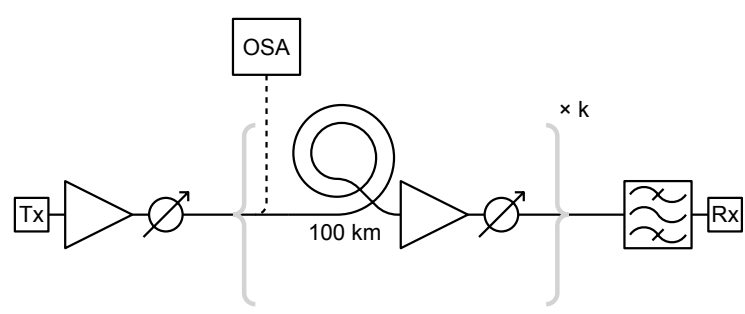

Fig. 1: Layout of the transmission system measurements. 


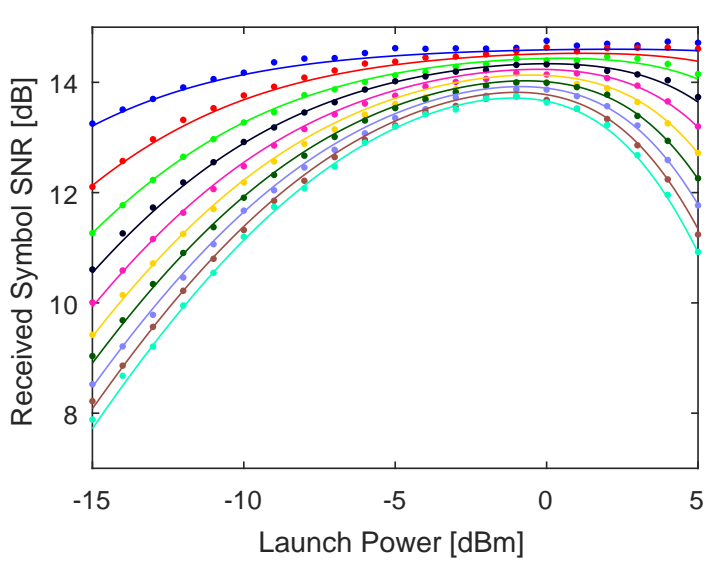

Fig. 2: Received symbol SNR as a function of launch power for transmission through 1 to 10 spans. Measured (points) and (lines) modeled based on the EGN model of NLI and a global three parameter fit.

span was maintained at the desired value, while the total power including ASE noise increases along the link.

The output of the 10th span was connected to the tunable optical channel filter to reduce the out of band ASE noise before being attenuated to a constant $-10 \mathrm{dBm}$ and connected to the receiver input. For the lowest launch power the receiver input power fell slightly to $-14.2 \mathrm{dBm}$ but this did not affect the receiver performance. The received SNR was calculated from the received signal using the moments based estimation technique $^{6}$. The transmission performance for shorter link lengths was measured by removing the last span each time. Then the whole process was repeated for the next desired launch power. The full set of measurements were repeated 4 times.

\section{Analysis and Results}

At each distance the measured SNR as a function of launch power was modeled by the three parameter model $^{7}$ given by

$$
S N R^{-1}=\left(\frac{P}{A S E+\eta P^{3}}\right)^{-1}+S N R_{0}^{-1}
$$

where $P$ is the launch power [mW], $A S E$ is the received ASE noise power in the receiver filter bandwidth [mW], $\eta$ is the nonlinear interference coefficient for the receiver filter bandwidth $\left[\mathrm{mW}^{-2}\right.$ ] and $S N R_{0}$ accounts for the transceiver internal noise.

The model parameters were found for each transmission distance by least squares fitting the inverse of the measure SNR, $S N R^{-1}$ against the launch power. Finally the three parameter model was globally optimized for the whole data set by including the distance dependence of the three
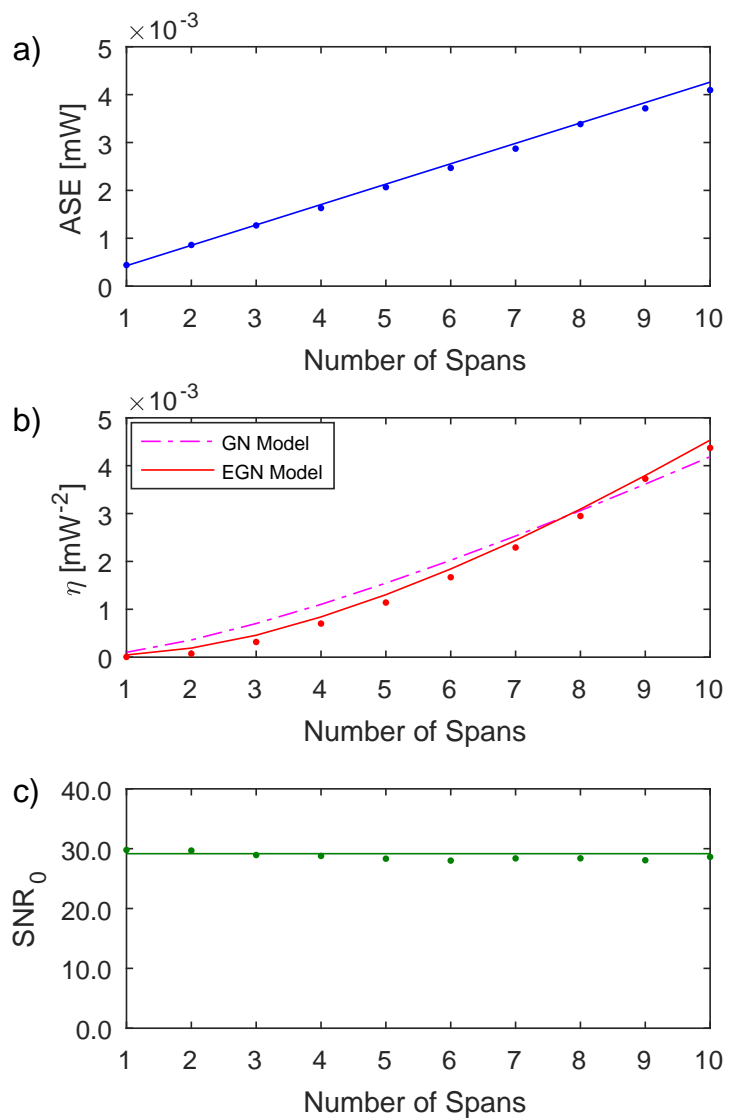

Fig. 3: The three parameters as a function of number of transmitted spans, a) ASE noise, b) NLI coefficient $\eta$ and c) transceiver noise. Points show the results of a three parameter fit to SNR vs launch power for each number of transmission spans, lines show the results of a global three parameter fit and the distance dependence in equation (2).

parameters as:

$$
\begin{aligned}
A S E(k) & =k \cdot A S E_{0} \\
\eta(k) & =\gamma^{2} f(k) \\
S N R_{0}(k) & =S N R_{0}
\end{aligned}
$$

where $k$ is the number of spans, $A S E_{0}$ is the average ASE noise [mW] in the receiver filter bandwidth per amplifier, $\gamma$ is the nonlinear coefficient $\left[\mathrm{W}^{-1} \cdot \mathrm{km}^{-1}\right]$ and $f(k)$ are pre-determined factors calculated by integration of the coherent GN or EGN model ${ }^{8,9}$ for $k$ spans. The transceiver SNR, $S N R_{0}$, was assumed to be independent of distance. The global parameters; $A S E_{0}, \gamma$ and $S N R_{0}$ where optimized by minimizing the sum of square residuals between the modeled and measured SNR in the log domain [dB]. Fitting the SNR in the log domain avoids the shorter distances and thus higher SNR unduly influencing the fitted parameters. The RMS fit residual was $0.07 \mathrm{~dB}$ for the EGN model case and $0.10 \mathrm{~dB}$ for the $\mathrm{GN}$ model case.

Fig. 2 shows the measured SNR (points) as a function of launch power for the 10 span lengths 


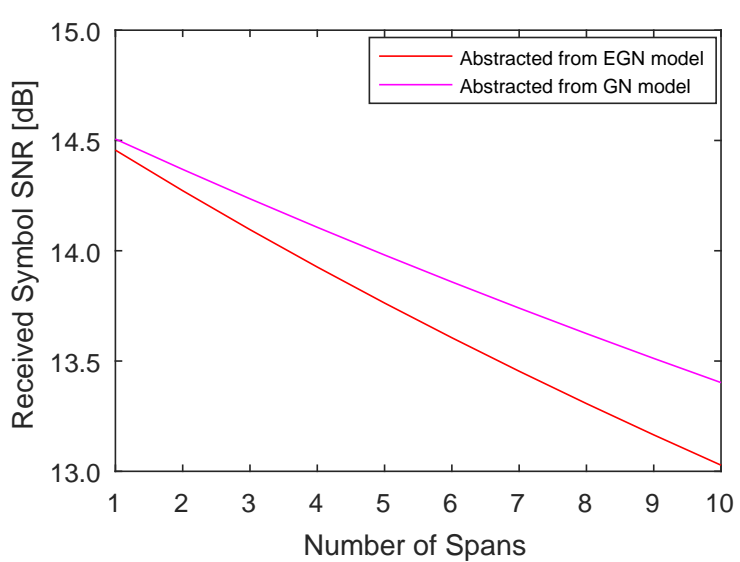

Fig. 4: Received symbol SNR vs number of transmission spans, for the measured single channel probe(points) and global three parameter model(lines) at optimum launch power and at the full load launch power $-4 \mathrm{dBm}$ compared to the predicted worst case full load SNR from the abstracted links.

and also the modeled SNR (lines) based on the EGN model of NLI and the globally fitted three parameters. Fig. 3 shows the individually fitted three parameters at each distance(points) and the model parameters (lines) based on the globally fitted parameters and equation (2). This results in $A S E_{0}=4.3 \times 10^{-4} \mathrm{~mW}$ equivalent to an amplifier noise figure of $4.6 \mathrm{~dB}, \gamma=1.14 \mathrm{~W}^{-1} \cdot \mathrm{km}^{-1}$ and a transceiver noise limited SNR, $S N R_{0}$ of 29.2. Using the GN model of NLI leads to an under estimation of the nonlinear coefficient, $\gamma=0.72 \mathrm{~W}^{-1} \cdot \mathrm{km}^{-1}$. It is seen in Fig. $3 \mathrm{~b}$ ) that the GN model does not fit the NLI coefficient well, while the EGN model gives a distance dependence that follows the individually fitted curves.

Finally we calculate the abstracted SNR degradation for a single span. The coherent $\mathrm{GN}$ model for the worst channel, the central channel, of a fully loaded link (80 channels of 11.5 GBaud on a $50 \mathrm{GHz}$ grid) over the longest path was divided by the number of spans to obtain the abstracted SNR degradation for a single span. Under these worst case condition the optimum launch power was found. Fig.4 shows the expected symbols SNR at the receiver for transmission through 1 to 10 spans and compares the results where the abstraction has used the EGN or GN model. This shows that the abstraction from the GN model over estimates performance as it under estimates the NLI. A error of approximately $0.4 \mathrm{~dB}$ in predicted performance occurs if the single channel probe is not used with the EGN model of NLI to accurately abstract the nonlinear coefficient.

\section{Conclusions}

We have shown that for single channel transmission the EGN model of NLI provides the best fit to the measured symbol SNR for a global three parameter model. The technique was applied to a laboratory network to probe the component properties and abstract the full load link parameters. The GN model based abstraction underestimates the nonlinear coefficient leading to an optimistic abstracted SNR by $0.4 \mathrm{~dB}$ at $1000 \mathrm{~km}$.

Future work will include confirming the abstraction under more loaded DWDM conditions and extending the work to an installed dark fiber network where the spans are of unequal length and the components remote. Consideration will also be given to adapt the technique for in service measurements.

\section{Acknowledgements}

The authors acknowledge the support of Ciena, the UK EPSRC project INSIGHT [EP/L026155/1] and the EU ICONE project grant \#608099.

\section{References}

[1] D. J. Ives, A. Alvarado, and S. J. Savory, "Throughput Gains From Adaptive Transceivers in Nonlinear Elastic Optical Networks," J. Light. Technol., Vol. 35, no. 6, pp. 1280-1289, (2017).

[2] R. Nejabati et al., "Optical network virtualization," Proc. ONDM, (2011).

[3] Y. Ou et al., "Optical Network Virtualization using Multi-technology Monitoring and Optical Virtualize-able Transceiver," Proc. OFC, W3F.7, Anaheim (2016).

[4] S. Yan et al., "Multilayer Network Analytics With SDNBased Monitoring Framework," J. Opt. Commun. Netw., Vol. 9, no. 2, pp. A271-A279, (2017).

[5] M. Bouda et al., "Accurate Prediction of Quality of Transmission with Dynamically Configurable Optical Impairment Model," Proc. OFC, Th1J.4, Los Angeles (2017).

[6] D. J. Ives et al., "Estimating OSNR of equalised QPSK signals," Opt. Express, Vol. 19, no. 26, pp. B661-B666, (2011).

[7] H. Chin et al., "Probabilistic Design of Optical Transmission Systems," J. Light. Technol., Vol. 35, no. 4, pp. 931940, (2017).

[8] P. Poggiolini, "The GN Model of Non-Linear Propagation in Uncompensated Coherent Optical Systems," J. Light. Technol., Vol. 30, no. 24, pp. 3857-3879, (2012).

[9] A. Carena et al., "EGN model of non-linear fiber propagation," Opt. Express, Vol. 22, no. 13, pp. 16335-16362, (2014). 